\title{
Self-reported use of evidence-based medicine and smoking cessation 6 - 9 months after acute coronary syndrome: A single-centre perspective
}

\author{
B Griffiths, ${ }^{1} \mathrm{MB} \mathrm{ChB} ;$ M Lesosky, ${ }^{1} \mathrm{PhD} ;$ M Ntsekhe, ${ }^{2} \mathrm{MD}, \mathrm{PhD}$ \\ ${ }^{1}$ Department of Medicine, Faculty of Health Sciences, University of Cape Town and Groote Schuur Hospital, Cape Town, South Africa \\ ${ }^{2}$ Division of Cardiology, Department of Medicine, Faculty of Health Sciences, University of Cape Town and Groote Schuur Hospital, \\ Cape Town, South Africa
}

Corresponding author: B Griffiths (bpgriffiths@gmail.com)

\begin{abstract}
Background. Good evidence exists to support the use of secondary prevention medications (aspirin, statins, beta-blockers and angiotensinconverting enzyme inhibitors (ACEIs) or angiotensin receptor blockers (ARBs)) and smoking cessation in patients after acute coronary syndromes (ACSs). Little is currently known about adherence to medication and smoking behaviour after discharge in South Africa.

Methods. We conducted a cross-sectional analysis of all patients with a diagnosis of ACS discharged from the Coronary Care Unit at Groote Schuur Hospital, Cape Town, between 15 November 2011 and 15 April 2012. Patients were telephoned 6 - 9 months after discharge and completed a standardised questionnaire detailing current medication use, reasons for non-adherence and smoking status.

Results. Prescribing of secondary prevention medications at discharge was high (aspirin $94.5 \%$, statins $95.7 \%$, beta-blockers $85.4 \%$, ACEIs/ ARBs $85.9 \%$ ), and $70.7 \%$ of patients were discharged on a combination of all four drugs. At 6 - 9-month follow-up, the proportion using these medications had dropped by $8.9 \%$ for aspirin, $10.1 \%$ for statins, $6.2 \%$ for beta-blockers and $17.9 \%$ for ACEIs/ARBs. Only $47.2 \%$ remained on all four drugs, a reduction of $23.5 \%$. Of the $56.0 \%$ of patients who were smokers, $31.4 \%$ had stopped smoking.

Conclusions. A significant decline in adherence to recommended therapy 6 - 9 months after discharge and a poor rate of smoking cessation suggest that efforts to educate patients about the importance of long-term adherence need to be improved. Furthermore, more effective interventions than in-hospital reminders about the hazards of smoking are needed to improve smoking cessation.
\end{abstract}

S Afr Med J 2014;104(7):483-487. DOI:10.7196/SAMJ.7798

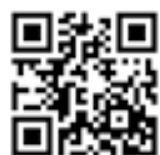

The incidence of ischaemic heart disease (IHD) is on the rise in Africa. Recent projections suggest that by 2030 IHD will become a leading cause of death on the continent, surpassing HIV/AIDS. ${ }^{[1]}$ In the South African National Burden of Disease Study in $2000,{ }^{[2]}$ IHD was found to be the largest single cause of death in the Western Cape Province (12\%), followed by stroke (8.8\%) and HIV/AIDS (8.4\%), and non-communicable diseases accounted for a larger proportion of deaths in the Western Cape (58\%) than in the rest of South Africa (SA) (38\%). In a subsequent study, ${ }^{\left[{ }^{[3]}\right.}$ mortality from IHD in Cape Town was found to be very similar to HIV/ AIDS (76 v. 83 deaths/100 000 population, respectively), and IHD featured as the leading cause of death from non-communicable diseases. The Second National Burden of Disease Study is nearing completion and will give us more current data on mortality trends. The epidemiological transition of cardiovascular disease described by Yusuf et al. ${ }^{[4]}$ suggests that with progression from underdevelopment to industrialisation we can expect an increasing burden of degenerative disease, particularly IHD and stroke.

The use of evidence-based optimal medical therapy (dual antiplatelet therapy, statins, beta-blockers and angiotensin-converting enzyme inhibitors (ACEIs) or angiotensin receptor blockers (ARBs)) after acute coronary syndrome (ACS) is known to reduce 6-month mortality by up to $87 \% \cdot{ }^{[5]}$ Patients who continue to smoke after an ACS have a significantly increased risk of a future acute myocardial infarction (MI) compared with those who quit. ${ }^{[6]}$ Furthermore, smoking in the presence of established IHD is associated with a significantly increased risk of death, including sudden cardiac death. Over an 8-year period, smoking cessation reduces this risk to levels comparable to that for people who have never smoked ${ }^{[7]}$ Despite this, medication adherence and smoking cessation in patients after discharge are often disappointing. ${ }^{[6-9]}$ Results from the Prospective Urban Rural Epidemiology (PURE) study ${ }^{[10]}$ suggest that in SA, and in countries with similar income profiles, use of these medicines in patients with coronary heart disease at a median follow-up period of 4 years varies between $21.1 \%$ and $31 \%$, compared with $46.5 \%$ and $70.9 \%$ for upper-income countries. These figures are even lower when Africa as a whole is compared with other areas. ${ }^{[10]}$ The reasons for discontinuation of medication are diverse, and include the high cost of obtaining medicine, high pill burden and lower level of education. ${ }^{[11]}$

To date there are no published SA data on the medium- to longterm ( $>6$ months) use of secondary prevention medication and smoking cessation among patients discharged from hospital after an ACS. We designed this cross-sectional study to assess the continued use of four selected drug classes and rates of smoking cessation at a follow-up period of 6 - 9 months post discharge after an ACS.

\section{Methods \\ Study design}

Cross-sectional analysis with retrospective subject identification. 


\section{Study population}

Groote Schuur Hospital is an 893-bed state-funded tertiary hospital in Cape Town, SA. It has a six-bed coronary care unit (CCU) and a cardiac catheterisation laboratory. Patients who are admitted to the CCU either present directly to the emergency unit or are referred from secondary-level institutions. Those who survive their acute cardiac insult are discharged home on optimal medical therapy and advised about smoking cessation. Clopidogrel, the only $\mathrm{P}_{2} \mathrm{Y}_{12}$ receptor antagonist available in our setting and now widely available, was only offered to those patients who underwent percutaneous coronary intervention during the study period, owing to funderinduced prescribing limitations at the time. Apart from a limited discussion about the hazards of smoking and its adverse effects on coronary artery disease, no specific smoking cessation guidance is offered. Long-term care and follow-up take place at the community health centre closest to the patient's home. Medication is supplied free of charge to patients who are pensioners, unemployed, or earn less than R36 000 per annum, which includes the vast majority of patients admitted to our unit.

We retrospectively enrolled all patients discharged alive from the CCU with a final diagnosis of unstable angina pectoris, non-ST elevation MI or ST-elevation MI during the 5-month period between 15 November 2011 and 15 April 2012. In each case the consultant cardiologist in charge of the patient's care made the diagnosis.

Approval to conduct the study was obtained from the Human Research Ethics Committee, Faculty of Health Sciences, University of Cape Town.

\section{Data collection}

A medical record review was performed and a standardised data capture form completed for each eligible patient. Information gathered included demographic characteristics, income group, comorbid conditions, medication use on admission, smoking status, ACS diagnosis, ACS in-hospital treatment, length of stay and discharge medication.

The principal author performed follow-up via a telephone call with the patient or primary caregiver at a time period between 6 and 9 months after discharge. A Xhosa-speaking interpreter performed three of the interviews under the guidance of the principal author, and a single patient was contacted by email. Verbal informed consent for enrolment into the study was obtained at this time from the patient or caregiver, and it was made clear that the telephone call was for study purposes only.

Patients were encouraged to fetch their current medications and actively list them over the telephone. Where a medication of interest was not listed, the patient was asked why this was the case. They were then asked a standardised smoking question: 'Do you smoke every day, only some days, or never?' Any reported smoking classified the patient as an ongoing smoker.

Interviews were conducted primarily in English, Afrikaans or Xhosa only being used if the patient was unable to communicate in English or requested otherwise, and the interviewer did not identify himself as a doctor unless specifically asked. Where behaviour hazardous to health was identified during the interview, such as ongoing smoking or non-adherence to medication, this was addressed prior to completing the interview.

\section{Statistical analysis}

Means and standard deviations were calculated for the full data and various data subsets. Comparisons of differences between groups were calculated using Fisher's exact test or the Wilcoxon rank-sum test (for categorical and continuous variables), as appropriate. A significance level of $p<0.05$ was used. All statistical analysis was done using R 3.0.

\section{Results \\ Patient characteristics}

A total of 164 patients with a diagnosis of ACS were discharged alive from the CCU and were therefore eligible for enrolment into the study. Their mean age was 58.6 years, and 97 (59.1\%) were male. The baseline demographic variables, type of ACS and inpatient treatment strategy are summarised in Table 1 .

Of the 164 patients enrolled in the study, 125 (76.2\%) completed follow-up interviews. Two patients (1.6\%) fell outside the planned follow-up range of 6 - 9 months (9.5 and 10.75 months), but were included for the sake of completeness and the low likelihood of two individuals making a difference to the results. Of the remaining patients, $19(11.6 \%)$ had died, $19(11.6 \%)$ were not contactable, and one $(0.6 \%)$ declined participation (Fig. 1). The patients who died or were lost to follow-up were more likely to have received medical treatment only $(p=0.01)$ and were significantly older $(p<0.0001)$.

\section{Medication use at discharge and at 6 - 9 months' follow-up}

Of the 164 patients enrolled in the study, 155 (94.5\%) were discharged on aspirin. Of the patients followed up $(n=125), 85.6 \%(n=107)$ remained on this medication, a reduction of $8.9 \%(p=0.01)$. Factors associated with ongoing aspirin use, by univariate analysis, were a previous diagnosis of IHD $(p=0.01)$ and having undergone coronary angiography $(p=0.0002)$. Reasons given for aspirin non-adherence were dyspepsia, iron deficiency anaemia, introduction of warfarin therapy, and not realising the importance of continued use.

Statins were prescribed to 157 patients (95.7\%) at discharge. At follow-up $85.6 \%(107 / 125)$ remained on this medication, a reduction of $10.1 \%$ ( $p=0.0008)$. The only factor associated with continued use was a diagnosis of hypercholesterolaemia $(p=0.02)$. Reasons given for discontinuation of statins were nausea, and not realising the importance of continued use.

Beta-blockers were prescribed for 140 patients (85.4\%) at discharge. At follow-up 79.2\% (99/125) remained on this medication, a reduction of $6.2 \%(p<0.0001)$. Factors associated with adherence were hypercholesterolaemia $(p=0.005)$, a previous diagnosis of IHD $(p=0.005)$, and female gender $(p=0.02)$. Reasons given for discontinuation were doctor-initiated cessation due to a reduced heart rate, and not realising the importance of continued use.

ACEIs/ARBs were prescribed for 141 patients (85.9\%) at discharge. At follow-up, 68.0\% (85/125) were still using this medication, the largest reduction in use for any of the four drug classes at $17.9 \%$ $(p<0.0001)$. Ongoing use was associated with a previous diagnosis of IHD $(p=0.04)$. Reasons given for discontinuation were angiooedema, cough, doctor-initiated cessation due to low blood pressure, and not realising the importance of continued use.

The number of patients discharged on a combination of all four drug classes was 116 (70.7\%). Of the 125 patients followed up, only $47.2 \%(n=59)$ remained on all four drugs, a reduction of $23.5 \%$ $(p=0.001)$. A total of five patients $(4.0 \%)$ had stopped all medications because they did not realise the importance of ongoing use. Results are summarised in Table 2.

\section{Smoking on admission and at 6 - 9 months' follow-up} Of the 125 patients who were followed up, $70(56.0 \%)$ had been active smokers on admission to hospital, and 22 of these had had stopped smoking by the time of the interview, representing a smoking cessation rate of $31.4 \%$. One patient had taken up 
Table 1. Demographic characteristics of patients enrolled, completing interviews and lost to follow-up

\begin{tabular}{|c|c|c|c|}
\hline Variable & $\begin{array}{l}\text { Enrolled } \\
(N=164)\end{array}$ & $\begin{array}{l}\text { Completed interview } \\
(N=125)\end{array}$ & $\begin{array}{l}\text { Lost to follow-up } \\
(N=39)\end{array}$ \\
\hline Age (yrs), mean (SD) & $58.6(11.2)$ & $57.0(10.9)$ & $63.8(11.0)$ \\
\hline Male, $n(\%)$ & $97(59.1)$ & $71(56.8)$ & $26(66.7)$ \\
\hline \multicolumn{4}{|l|}{ Income category, $n(\%)$} \\
\hline Unemployed/pensioner & $47(28.6)$ & $31(24.8)$ & $16(41.0)$ \\
\hline Income <R36 000 p.a. & $85(51.8)$ & $66(52.8)$ & $19(48.7)$ \\
\hline Income <R72 000 p.a. & $14(8.5)$ & $14(11.2)$ & - \\
\hline Income >R72 000 p.a. & $13(7.9)$ & $9(7.2)$ & $4(10.3)$ \\
\hline Private medical aid/foreigner & $4(2.4)$ & $4(3.2)$ & - \\
\hline Unknown & $1(0.6)$ & $1(0.8)$ & - \\
\hline \multicolumn{4}{|l|}{ Medical history, $n(\%)$} \\
\hline Hypertension & $99(60.3)$ & $71(56.8)$ & $28(71.8)$ \\
\hline Diabetes mellitus & $51(31.0)$ & $36(28.8)$ & $15(38.5)$ \\
\hline Hypercholesterolaemia & $73(44.5)$ & $57(45.6)$ & $16(41.0)$ \\
\hline IHD & $83(50.6)$ & $61(48.8)$ & $22(56.4)$ \\
\hline Current smoker, $n(\%)$ & $90(54.8)$ & $70(56.0)$ & $20(51.3)$ \\
\hline \multicolumn{4}{|l|}{ Diagnosis, $n(\%)$} \\
\hline ST-elevation MI & $59(35.9)$ & $44(35.2)$ & $15(38.5)$ \\
\hline Non ST-elevation MI & $77(46.9)$ & $57(45.6)$ & $20(51.3)$ \\
\hline Unstable angina pectoris & $28(17.0)$ & $24(19.2)$ & $4(10.3)$ \\
\hline \multicolumn{4}{|l|}{ Treatment, $n(\%)$} \\
\hline Medical only & $95(57.9)$ & $65(52.0)$ & $30(76.9)$ \\
\hline PCI & $59(35.9)$ & $50(40.0)$ & $9(23.1)$ \\
\hline Coronary artery bypass graft & $10(6.09)$ & $10(8.0)$ & - \\
\hline Angiogram (including PCI), $n(\%)$ & $112(68.2)$ & $92(73.6)$ & $20(51.3)$ \\
\hline Length of stay (days), mean (SD) & $3.018(1.9)$ & $2.98(2.0)$ & $3.13(1.7)$ \\
\hline
\end{tabular}

smoking for the first time after discharge. The only factor independently associated with an increased rate of smoking cessation, by univariate analysis, was a longer length of hospital stay $(p=0.006)$. We could find no relationship between smoking cessation rate and age, gender, income category, comorbid conditions, admission diagnosis or treatment received.

\section{Discussion}

There was good early initiation of secondary prevention medication after ACS in our cohort of patients. It has been shown that patients who do not start medication shortly after the acute event are unlikely to ever have medication added, and a focused effort to start treatment in the immediate postinfarction period is likely to provide longterm benefit. ${ }^{[12]}$ When we compare these findings with those of the Clinical Pathways for Acute Coronary Syndromes in China
$(\mathrm{CPACS})^{[8]}$ and the Global Registry of Acute Coronary Events (GRACE) ${ }^{[9]}$ studies, our prescribing rates for the four selected drugs at discharge after ACS are higher for all drug classes (Table 3 and Fig. 2). Since both the above studies used the same method of follow-up as we did, namely telephone calls and active listing of medications, they provide a reasonable comparison. The CPACS investigators felt that there was a significant gap between evidence and practice in medication prescribing after ACS, which was not found in our study. It should be noted that the low rates of prescribing of secondary prevention medication found in the GRACE study may in part be because the data were collected from 1999 to 2003, when less emphasis was placed on these medications. Our finding of a high early initiation rate of all four recommended drugs is important and demonstrates that adherence to best-practice guidelines in our setting is feasible.

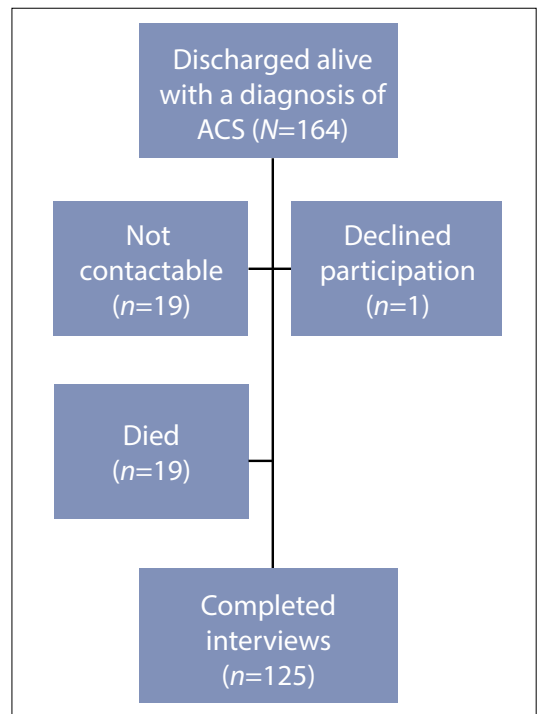

Fig. 1. Flow diagram demonstrating followup of enrolled patients. (ACS = acute coronary syndrome.) 
Table 2. Medication use at discharge and follow-up

\begin{tabular}{|c|c|c|c|c|}
\hline & Discharge $(N=164), n(\%)$ & Follow-up $(N=125), n(\%)$ & Reduction in use (\%) & $p$-value \\
\hline Aspirin & $155(94.5)$ & $107(85.6)$ & 8.9 & 0.01 \\
\hline Statin & $157(95.7)$ & $107(85.6)$ & 10.1 & 0.0008 \\
\hline Beta-blocker & $140(85.4)$ & $99(79.2)$ & 6.2 & $<0.0001$ \\
\hline ACEI/ARB & $141(85.9)$ & $85(68.0)$ & 17.9 & $<0.0001$ \\
\hline Four-drug comb. & $116(70.7)$ & $59(47.2)$ & 23.5 & 0.001 \\
\hline
\end{tabular}

Table 3. Comparison between medication use at discharge and follow-up in the present study and two similar studies

\begin{tabular}{|c|c|c|c|c|c|c|c|c|c|}
\hline & \multicolumn{3}{|c|}{ Griffiths et al. (SA, present study) } & \multicolumn{3}{|c|}{ Bi et al. ${ }^{[8]}$ (CPACS China) } & \multicolumn{3}{|c|}{ Eagle et al. ${ }^{[9]}$ (GRACE multi-country) } \\
\hline & $\begin{array}{l}\text { DC } \\
(N=164) \\
n(\%)\end{array}$ & $\begin{array}{l}\text { FU } \\
(N=125) \\
n(\%)\end{array}$ & Diff., \% & $\begin{array}{l}\mathrm{DC} \\
(N=2901) \\
n(\%)\end{array}$ & $\begin{array}{l}\text { FU } \\
(N=2521) \\
n(\%)\end{array}$ & Diff., \% & $\begin{array}{l}\text { DC } \\
(N=13830) \\
n(\%)\end{array}$ & $\begin{array}{l}\text { FU } \\
(N=13830) \\
n(\%)\end{array}$ & Diff., \% \\
\hline Aspirin & $\begin{array}{l}155 \\
(94.5)\end{array}$ & $107(85.6)$ & -8.9 & $\begin{array}{l}2687 \\
(92.7)\end{array}$ & $\begin{array}{l}2211 \\
(88.2)\end{array}$ & -4.5 & $\begin{array}{l}12463 \\
(90.1)\end{array}$ & $\begin{array}{l}11465 \\
(82.9)\end{array}$ & -7.2 \\
\hline $\begin{array}{l}\text { Lipid-lowering } \\
\text { agent }\end{array}$ & $\begin{array}{l}157 \\
(95.7)\end{array}$ & $107(85.6)$ & -10.1 & $\begin{array}{l}2332 \\
(80.4)\end{array}$ & $\begin{array}{l}1654 \\
(65.8)\end{array}$ & -14.6 & $\begin{array}{l}6320 \\
(45.7)\end{array}$ & $\begin{array}{l}5522 \\
(39.9)\end{array}$ & -6.1 \\
\hline Beta-blocker & $\begin{array}{l}140 \\
(85.4)\end{array}$ & $\begin{array}{l}99 \\
(79.2)\end{array}$ & -6.2 & $\begin{array}{l}2031 \\
(70.0)\end{array}$ & $\begin{array}{l}1798 \\
(71.8)\end{array}$ & +1.8 & $\begin{array}{l}7738 \\
(56.0)\end{array}$ & $\begin{array}{l}6796 \\
(49.1)\end{array}$ & -6.9 \\
\hline ACEI/ARB & $\begin{array}{l}141 \\
(85.9)\end{array}$ & $\begin{array}{l}85 \\
(68.0)\end{array}$ & -17.9 & $\begin{array}{l}2194 \\
(75.6)\end{array}$ & $\begin{array}{l}1694 \\
(67.2)\end{array}$ & -8.4 & $\begin{array}{l}2379 \\
(17.2)\end{array}$ & $\begin{array}{l}1906 \\
(13.8)\end{array}$ & -3.4 \\
\hline Four-drug comb. & $\begin{array}{l}116 \\
(70.7)\end{array}$ & $\begin{array}{l}59 \\
(47.2)\end{array}$ & -23.5 & $\begin{array}{l}1384 \\
(47.7)\end{array}$ & $\begin{array}{l}1088 \\
(43.6)\end{array}$ & -4.1 & NA & NA & NA \\
\hline
\end{tabular}

We found a significant increase in the rate of non-adherence to the selected medications in our group of patients beyond 6 months. Medication cessation for appropriate reasons, such as ACEI-induced angio-oedema or betablocker-induced symptomatic bradycardia, is unavoidable and expected for a minority of patients. What is more concerning is that patients stopped medication because they did not realise the importance of ongoing use, which was found to be a reason for nonadherence for each of the four drug classes, and for the five patients who stopped all four drugs completely. Rates of discontinuation of medication were higher for most of the drug classes when compared with the CPACS and GRACE studies (Table 3 and Fig. 2), the exceptions being lipid-lowering agents when compared with CPACS (a 10.1\% drop-off in adherence v. 14.6\%) and beta-blockers when compared with GRACE $(6.2 \%$ v. $6.9 \%)$. It is not clear from our data why the rates of non-adherence are higher. There is no clear link to a language barrier, as all but three of the patients could speak English or Afrikaans, both of which are spoken in the CCU by doctors and nursing staff. There was also no demonstrable link between income

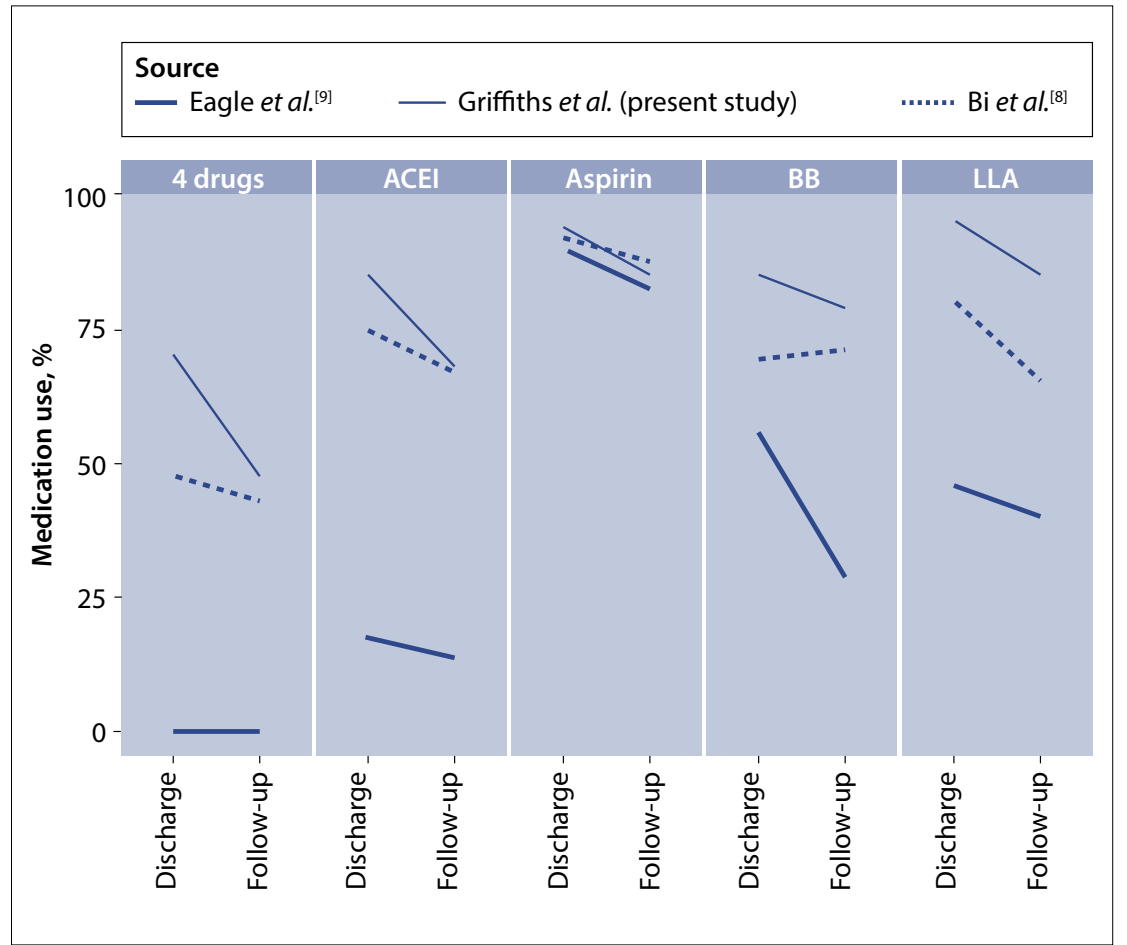

Fig. 2. Graphic representation of the comparison between medication use at discharge and follow-up in the present study and two similar studies. (ACEI = angiotensin-converting enzyme inhibitor; $B B=$ beta-blocker; $L L A=$ lipid-lowering agent.) 
category and adherence, and SA and China are both rated as upper middle-income countries by the World Bank Country Classification, so adherence rates based purely on income would be expected to be similar. It is possible that level of education and insight into disease could play a role, but these were not included in our questionnaire. No patient mentioned a shortage of medication at a local clinic as a reason for discontinuation. The frequent reporting of non-adherence to medication as a result of not understanding the importance of ongoing use implies that good communication with the patient during the index hospital admission, with a focus on education about the disease and future treatment, is essential. Enhanced communication between treating doctor and patient, as well as involving ancillary healthcare providers such as nursing staff and pharmacists, have both been shown to improve adherence, both during admission and through community-based cardiac rehabilitation programmes. Innovative measures such as the use of short message service (SMS) reminder technology for mobile phones, which has been demonstrated to improve adherence to antiretroviral medication in SA and to other chronic medications elsewhere, should be looked at to improve adherence in our setting.

More of our patients continued to smoke after an ACS than has been found in similar studies. Evidence from the PURE study shows a clear link between smoking cessation after a coronary heart disease or stroke event and country income category, with cessation rates highest in upper-income countries, and decreasing with declining income category of the country. ${ }^{[13]}$ The smoking cessation rate in upper middle-income countries such as SA was found to be $54.6 \%{ }^{[13]}$ which is still higher than the $31 \%$ rate in our group. We did not ask patients whether they had tried to stop smoking, or indeed whether they wanted to try to stop, so the rate of failed attempts at smoking cessation is unknown. The Western Cape is known to have the highest smoking rate of all the SA provinces, at $44.7 \%$ of men and $27 \%$ of women. ${ }^{[14]} \mathrm{A}$ recent Cochrane review found that intensive behavioural interventions in hospitalised patients, followed by at least a month of outpatient supportive contact, was effective at significantly improving smoking cessation rates, and it should be considered as an option in this group of patients. The addition of nicotine replacement therapy in gum or patch form further improves smoking cessation. At present there is not sufficient evidence to prove that bupropion or varenicline in addition to intensive counselling in hospitalised patients increases cessation rates over intensive counselling alone. ${ }^{[15]}$ The smoking cessation rates in our cohort suggest that adoption of these and other evidence-based methods of influencing smoking behaviour should be made an urgent priority.

\section{Study limitations}

Our study has a number of limitations. We measured adherence by self-report, with no electronic or pill count data to back up our findings. Although this method of active listing of medications over the telephone tends to overestimate adherence, it has been used successfully in similar studies ${ }^{[8,9,11]}$ and is highly specific. While measurement of smoking by self-report can lead to an overestimation of smoking cessation, this method has been shown to correlate well with cotinine testing in most studies. Other limitations include enrolling patients from only a single urban tertiary centre, having a relatively small sample size, and borderline significance of univariate analyses. Not knowing the medication adherence and smoking cessation rates of those patients who died is another limitation, as this information may have improved insight into mortality in this group of patients. These factors raise the concern that our findings may not be representative of the population as a whole. In order to avoid selection bias at enrolment, we included every patient with ACS discharged alive from the CCU over the selected 5-month period, and did not limit inclusion to those patients who were followed up at our institution. SA is a diverse country with broad variations in culture, income and access to healthcare, and a similar multicentre, prospective study in future could improve our understanding of the challenges posed by medication adherence and smoking cessation after ACS

\section{Conclusion}

This study has provided valuable insight into prescribing practices, medium-term adherence patterns after ACS, and smoking cessation rates beyond 6 months, areas for which there are currently no published data in our setting. Three findings were of particular importance: (i) we found that our prescribing of secondary prevention medication at discharge is high ( $70.7 \%$ for the four-drug combination); (ii) we noted that a fairly large proportion of our patients are discontinuing medication within a 6 -9-month period $(23.5 \%$ for the four-drug combination); and (iii) we demonstrated that a large proportion $(68.6 \%)$ of patients who were active smokers on admission to hospital continued to smoke, giving a cessation rate of $31.4 \%$. Given the importance of smoking cessation in improving short- and long-term outcomes, more focused strategies to improve this adverse behaviour, particularly in this high risk population, are required.

\section{References}

1. Ntsekhe M, Damasceno A. Recent advances in the epidemiology, outcome, and prevention of myocardial infarction and stroke in sub-Saharan Africa. Heart 2013;99(17):1230-1235. [http://dx.doi. roc/10.1136/hertint-2012-303585

2. Bradshaw D, Nannan N, Laubscher R, et al. South African National Burden of Disease Study 2000. Bradshaw D, Nannan N, Laubscher R, et al. South African National Burden of Disease Study 2000.
Estimates of Provincial Mortality: Western Cape Province. Cape Town: South African Medical Estimates of Provincial Mortality: Western Cape Province. Cape Town: South African Medical
Research Council, 2003. http://www.mrc.ac.za/bod/westerncape.pdf (accessed 26 November 2013). Research Council, 2003. http://www.mrc.ac.za/bod/westerncape.pdf (accessed 26 November 2013).
3. Groenewald P, Bradshaw D, Daniels J, et al. Cause of Death and Premature Mortality in Cape Town, 2001-2004. Cape Town: South African Medical Research Council, 2007. http://www.mrc.ac.za/bod/ capetownreport.pdf (accessed 26 November 2013).

4. Yusuf S, Reddy S, Ounpuu S, Anand S. Global burden of cardiovascular diseases. Part I: General considerations, the epidemiologic transition, risk factors, and impact of urbanisation. Circulation 2001;104(22):2746-2753. [http://dx.doi.org/10.1161/hc4601.099487]

5. Mukherjee D, Fang J, Chetcuti S, Moscucci M, Kline-Rogers E, Eagle KA. Impact of combination evidence-based medical therapy on mortality in patients with acute coronary syndromes. Circulation 2004;109(6):745-749. [http://dx.doi.org/10.1161/01.CIR.0000112577.69066.CB]

6. Chow CK, Jolly S, Rao-Melacini P, Fox KA, Anand SS, Yusuf S. Association of diet, exercise, and smoking modification with risk of early cardiovascular events after acute coronary syndromes.
smo Circulation 2010;121(6):750-758. [http://dx.doi.org/10.1161/circulationaha.109.891523]

7. Goldenberg I, Jonas M, Tenenbaum A, et al. Current smoking, smoking cessation, and the risk of 7. Goldenberg I, Jonas M, Tenenbaum A, et al. Current smoking, smoking cessation, and the risk of
sudden cardiac death in patients with coronary artery disease. Arch Intern Med 2003;163(19):2301sudden cardiac death in patients with coronary artery
2305. [http://dx.doi.org/10.1001/archinte.163.19.2301]

8. Bi Y, Gao R, Patel A, et al. Evidence-based medication use among Chinese patients with acute coronary syndromes at the time of hospital discharge and 1 year after hospitalization: Results from the Clinical Pathways for Acute Coronary Syndromes in China (CPACS) study. Am Heart J 2009;157(3):509-516. [http://dx.doi.org/10.1016/j.ahj.2008.09.026]

9. Eagle KA, Kline-Rogers E, Goodman SG, et al. Adherence to evidence-based therapies after discharge for acute coronary syndromes: An ongoing prospective, observational Study. Am J Med 2004;117(2):73-81. [http://dx.doi.org/10.1016/j.amjmed.2003.12.041]

10. Yusuf S, Islam S, Chow CK, et al. Use of secondary prevention drugs for cardiovascular disease in the community in high-income, middle-income, and low-income countries (the PURE study): A prospective epidemiological survey. Lancet 2011;378(9798):1231-1243. [http://dx.doi.org/10.1016/ prospective epidemiologi

11. Melloni C, Alexander KP, Ou FS, et al. Predictors of early discontinuation of evidence-based medicine after acute coronary syndrome. Am J Cardiol 2009;104(2):175-181. [http://dx.doi.org/10.1016/j. amicard.2009.03.013]

12. Gislason GH, Rasmussen IN, Abildstom SZ, et al. Long-term compliance with beta-blockers, angiotensin-converting enzyme inhibitors, and statins after acute myocardial infarction. Eur Heart $\mathrm{J}$ 2006;27(10):1153-1158. [http://dx.doi.org/10.1093/eurheartj/ehi705]

13. Teo K, Lear S, Islam S, et al. Prevalence of healthy lifestyle among individuals with cardiovascular disease in high-, middle- and low-income countries: The Prospective Urban Rural Epidemiology (PURE) study. JAMA 2013;309(15):1613-1621. [http://dx.doi.org/10.1001/jama.2013.3519]

14. Chopra M, Steyn N, Lambert V. Western Cape Burden of Disease Reduction Project: Decreasing the burden of cardiovascular disease. Final report 2007. http://www.westerncape.gov.za/text/2007/6/ cd_volume_6_cardiovascular_diseases.pdf (accessed 26 November 2013).

15. Rigotti NA, Clair C, Munafò MR, Stead LF. Interventions for smoking cessation in hospitalised patients. Cochrane Database Syst Rev 2012; Issue 5. Art. No.: CD001837. [http://dx.doi.org/10.1002/14651858. CD001837.pub3] 